\title{
TAROGE-M: Radio Observatory on Antarctic High Mountain for Detecting Near-Horizon Ultra-High Energy Air Showers
}

\section{Shih-Hao Wang* on behalf of the TAROGE and ARIANNA Collaboration}

(a complete list of authors can be found at the end of the proceedings)

${ }^{a}$ Department of Physics and Leung Center for Cosmology and Particle Astrophysics, National Taiwan University, No. 1, Sec. 4, Roosevelt Rd., Taipei 10617, Taiwan, R.O.C.

E-mail: wsh4180@gmail.com

The TAROGE-M observatory is an autonomous antenna array on the top of Mt. Melbourne ( $2700 \mathrm{~m}$ altitude) in Antarctica, designed to detect radio pulses from ultra-high energy (over $10^{17} \mathrm{eV}$ ) air showers coming from near-horizon directions. The targeted sources include cosmic rays, Earth-skimming tau neutrinos, and most of all, the anomalous near-horizon upward-going events of yet unknown origin discovered by ANITA experiments. The detection concept follows that of ANITA: monitoring large area of ice from high-altitude and taking advantage of strong geomagnetic field and quiet radio background in Antarctica, whereas having significantly greater livetime and scalability.

The TAROGE-M station, upgraded from its prototype built in 2019, was deployed in January 2020, and consists of 6 log-periodic dipole antennas pointing horizontally with bandwidth of 180-450 MHz. The station is then calibrated with drone-borne transmitter, with which the event reconstruction obtained $\sim 0.3^{\circ}$ angular resolution. The station was then smoothly operating in the following month, with the live time of $\sim 30$ days, before interrupted by a power problem, and its online filtering has identified several candidate cosmic-ray events and sent out via satellite communication. In this paper, the instrumentation of the station for polar and high-altitude environment, its radio-locating performance, the preliminary result on cosmic-ray detection, and the future extension plan are presented.

$37^{\text {th }}$ International Cosmic Ray Conference (ICRC 2021)

July 12 th - 23rd, 2021

Online - Berlin, Germany

\footnotetext{
${ }^{*}$ Presenter
} 


\section{Introduction}

The origin and characteristics of ultra-high-energy (UHE, above $10^{18} \mathrm{eV}$ ) cosmic rays and neutrinos have been long-standing puzzles. An effective way to observe UHE cosmic rays is to detect the radio pulses emitted by the extensive air showers they initiate, mainly through the geomagnetic emission created when electrons and positrons in air shower are deflected under the geomagnetic field. The radio detection technique has been established over past two decades, capable of inferring parameters of primary particles such as direction, energy and composition with good precision (see [1] for a review). The technique can also be applied to detecting air showers initiated by the delay of tau lepton produced when UHE Earth-skimming tau neutrinos interact with the rock via charged-current interaction. A radio observatory at high elevation can monitor large volume of Earth's crust as neutrino target, and hence a efficient detector for both UHE cosmic rays and tau neutrinos. The ANITA experiment [2,3], a stratospheric balloon-borne antenna array using 200-1000 MHz frequency band in Antarctica, has finished four flights and observed not only dozens of cosmic rays but also 4 upward-going anomalous events with tau-neutrino like signature, which origin calls for further confirmation. However, the short live time of balloon experiment typically of a month makes it harder to increasing the statistics significantly. As an alternative, locating radio detectors at high mountains $[4,5]$ with clear view of the horizon, though with less detection volume, can have lower energy threshold and much greater live time and scalability, and thus can obtain competitive sensitivity and beyond relatively easily in principle. Antarctic mountains are ideal places for radio detection of near-horizon UHE air showers for the strong $(>60 \mu \mathrm{T})$ and nearly vertical geomagnetic field and the quiet radio-frequency (RF) background. Therefore, we built an prototype antenna station in 2019 [4] on the top of Mt. Melbourne $\left(74^{\circ} 20^{\prime} 55.88^{\prime \prime} \mathrm{S}, 164^{\circ} 41^{\prime} 35.38^{\prime \prime}\right.$ E), an Antarctic volcano of $2720 \mathrm{~m}$ high, where the site is accessible with helicopter from Korean Jang Bogo (JBG) research station $30 \mathrm{~km}$ away at southwest. In this paper, the upgrade and the development since then are reported, and as a demonstration of air-shower detection, a preliminary search for UHE cosmic-ray signals is also presented.

\section{TAROGE-M Station Design and Operation Summary}

The first TAROGE ${ }^{1}$ antenna station on Mt. Melbourne (TAROGE-M, Fig. 1a) was upgraded from its prototype and deployed in January 2020. The station is designed for detecting radio pulses from ultra-high energy air showers coming from near-horizon directions, based on the experiences of TAROGE stations on mountains in Taiwan [6] and ARIANNA-HCR (hereafter HCR) station in Antarctica [7]. The receiver consists of 6 log-periodic dipole antennas (LPDA) pointing to the horizon with $7 \mathrm{dBi}$ gain above $180 \mathrm{MHz}$, with four of antennas horizontally polarized (Hpol) to align with the polarization of geomagnetic emission, one vertically polarized (Vpol) for the polarization measurement, and one also Hpol but pointing to opposite direction for vetoing any potential noise from other automatic stations ${ }^{2}$ behind several hundred meters away. The received signal is then passed through 180-450 MHz band-pass filter, a notch filter at $360 \mathrm{MHz}$, and two cascaded low-

\footnotetext{
${ }^{1}$ TAiwan Radio Observatory for Geomagnetic Emission [6], an acronym named after Taroko national park featuring mountains and gorges nearby the stations in eastern Taiwan.

2including a telecommunication, a weather, and a seismic stations.
} 


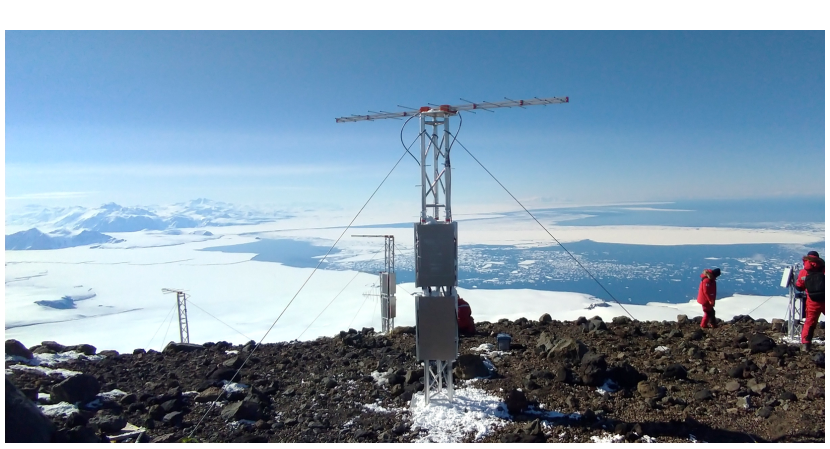

(a)

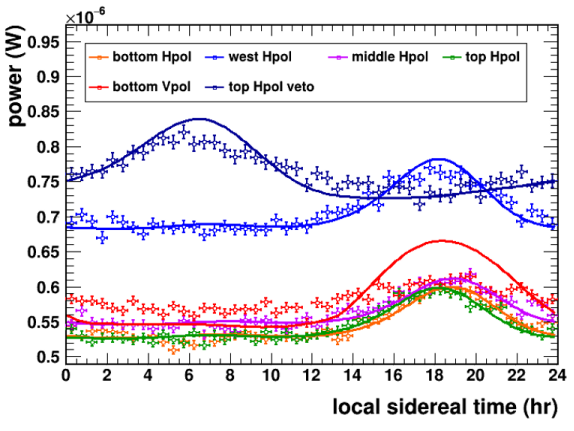

(b)

Figure 1: Left: TAROGE-M station on the top of Mt. Melbourne in Antarctica. Hpol antennas are mounted on $3 \mathrm{~m}$ tower, pointing to northern horizon, with $\sim 8.5 \mathrm{~m}$ separation between lower three towers, and $\sim 19 \mathrm{~m}$ between top (with veto antenna) and bottom ones, whereas the Vpol one is placed under the east Hpol.

Right: Markers and error bars indicate the mean and the error of mean, respectively, of the distribution of received power at $190-240 \mathrm{MHz}$ band for each channel, with total 21, 919 forced-trigger events, as function of local sidereal time. Curves are the simulated Galactic noise with a fitting of receiver noise power for constant offset. The distribution of veto channel is out of phase from others because of its opposite pointing to the back side.

noise amplifiers (LNA) with total $64 \mathrm{~dB}$ gain. The receiver bandwidth is chosen based on previous RF background survey in 2019 for suppressing narrow-band noise. The data acquisition (DAQ) is done by a 8-channel SST board [8] same as those used in ARIANNA experiment [9], which performance has been proven with years of operation at Ross Ice Shelf and South Pole. The SST board is set to $1 \mathrm{GHz}$ sampling rate, and storing 256 samples with 12-bits dynamic range for each channel. A trigger is formed by two stages: the channel-level trigger requires that the received waveform passes both positive and negative thresholds within $5 \mathrm{~ns}$, and then the station-level one requires 3-out-of- 4 coincidence of Hpol channel triggers within $32 \mathrm{~ns}$ for the signals to be digitized and recorded. An additional real-time narrow-band noise rejection is applied by computing the ratio of the peak magnitude of the FFT spectrum to the sum of the rest of spectrum, and an event is discarded if the ratio exceeds a given threshold of 0.3 at any non-veto channel. A single-board computer (BeagleBone Black) is added for online filtering (more details in Sec. 4.2) SST data to prioritize Hpol-dominant impulsive events to be transferred back to the server at northern hemisphere via Inmarsat satellite communication with limited data size. The computer also monitors the entire system, and can be remotely accessed and configured.

The system, with total power consumption less than $20 \mathrm{~W}$, is powered by eight $30 \mathrm{~W}$ solar photo-voltaic panels and $150 \mathrm{Ah}$ sealed lead-acid batteries, designed for operation throughout austral summer from August to April, and a wind turbine was also installed for investigating the feasibility of extended operation in winter. All electronics devices are contained in RF shielding boxes inside a thermally insulated enclosure on the ground, which keeps the internal temperature at $10-30{ }^{\circ} \mathrm{C}$ during operation, roughly $50^{\circ} \mathrm{C}$ higher than the ambient temperature.

The station was continuously operating since the deployment on Jan 25 , and the battery charging was stopped after Feb 21, probably due to sunlight got blocked by icing accumulated on solar panels during snow storm, causing the system ran out of power on Feb 24. However, the system failed in 
cold start, leading to total live time of about 30 days, and the problem was later reproduced in the lab test and identified as malfunctioning DC-to-DC converter under low temperature.

During operation, the trigger threshold was adjusted between 4 to 7 times of RMS voltage (typically $15 \mathrm{mV}$ ), resulting in typical event rate of $10^{-2}-10^{-3} \mathrm{~Hz}$. There are periods of high event rate of $1-10 \mathrm{~Hz}$ with each one lasting for a few hours to a few days, correlated with bad weather on the mountain, a similar phenomenon as observed by HCR station [7]. These "high-wind" events during these periods account for the majority of impulsive events in TAROGE-M data.

In 2020-2021 season, the hardware of the station was inspected and no major damage was found over the winter, but because of the halt in scientific research at JBG station due to COVID-19 pandemic, the DAQ system was taken back to lab for maintenance, upgrade and accessing full data.

\section{In-Situ Calibration}

The source direction of an event is an important discriminator for neutrino and cosmic-ray signals, and is reconstructed by the time difference of arrival (TDOA) of signals between Hpol antennas, following an interferometric method based on waveform cross-correlation, similar to that in [10]. Therefore, the calibration of antenna positioning and response of each channel is crucial for locating the sources. The antenna station was fast surveyed with photogrammetry and georeferenced by differential GPS (DGPS) right after the deployment. The photos are post-processed with Pix4DMapper software [11] to generate 3D station model, and a positioning precision better than $1 \mathrm{~cm}$ is obtained.

\subsection{Calibration with Galactic noise}

To monitor the radio-frequency (RF) background, forced trigger at every 100 seconds was enabled to obtain unbiased noise samples throughout the operation. The Galactic noise variation in these event can sever as in-situ calibration of receiver response, with analysis as in [12]. As shown in Fig. 1b, the received power in $190-240 \mathrm{MHz}$ band of all channels vary periodically with cycle of a sidereal day. This is consistent with the simulation consisting of Galactic noise modelled with LFMap [13] combining the antenna response in free space modelled with HFSS [14], measured electronics response, and a fitting of receiver noise power for constant power offset. Except the power of Vpol channel is less undulating than the expected one, which suggests antenna response got modified due to adjacent $\mathrm{Hpol}$ antenna on its top, the overall agreement between observation and expectation shows the modelled response is reliable for further analysis.

\subsection{Calibrating event reconstruction with drone-borne pulser}

A drone-borne calibration pulser system was developed [15] to calibrate and measure the performance of TAROGE-M event reconstruction, especially at near-horizon directions which are otherwise difficult to access and scan by other means. The drone pulser consists of a pulser module which pulse strength is configurable for stepped scan, a broadband Hpol biconical transmitter antenna, a DPS module with centimeter positioning accuracy, and a logger recording the pulsing timestamp, strength and position. Two drone flights were conducted on Jan 30, making grid scan at a distance of $\sim 500 \mathrm{~m}$ in front of the station (Fig. 2), with each flight lasting for about 15 minutes. 

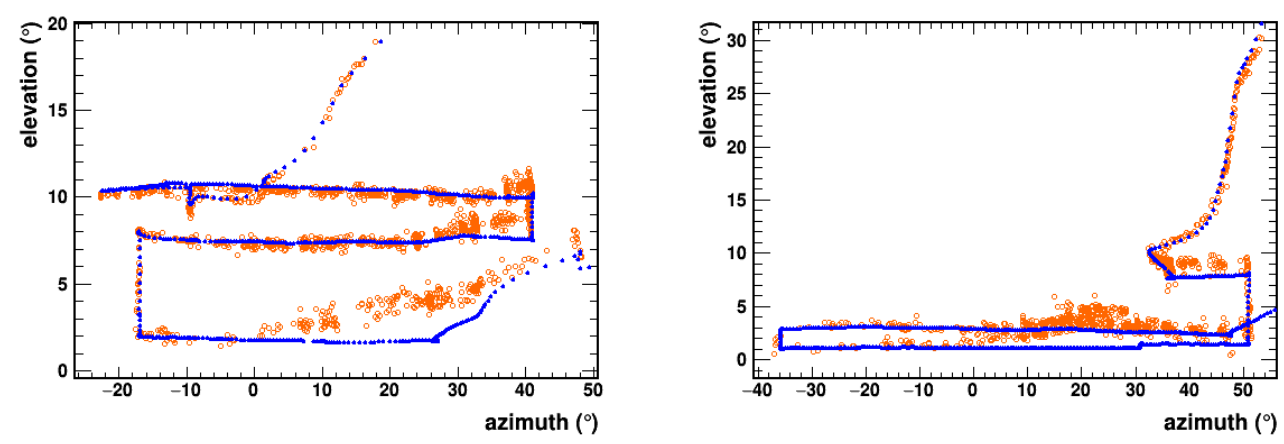

Figure 2: expected track (blue) of first (left) and second (right) drone pulser flights recorded by DGPS and the reconstructed one (orange) after calibration. The elevation angle is $0^{\circ}$ in horizontal direction, and azimuth angle is defined as $0^{\circ}$ at geographic north and positive to west, where LPDAs point to $\sim 10^{\circ}$.

The pulser was set to $5 \mathrm{~Hz}$ pulsing rate and $7 \mathrm{~dB}$ power dynamic range for characterizing trigger efficiency, and there are nearly 6000 pulser events recorded by the station.

The station orientation, the internal delay of receivers, and the phase center of LPDA antennas are calibrated by minimizing the squared sum of the difference between the observed (crosscorrelated) TDOA and the expected one over all selected events. A set of 593 high-quality pulser events are selected as calibration samples, whereas the rest of events are used to verify the result. The selected events have high signal-to-noise ratio (SNR), with position within the main lobe of receiver antennas where their responses are not much different, and at elevation angle $>3^{\circ}$, below which the effect of reflected signal from the ground becomes not negligible.

The event reconstruction proceeds as following: waveforms are first band-pass filtered at $180-330 \mathrm{MHz}$ and interpolated by a factor of 20 . Then waveforms have both the receiver and the transmitter responses deconvolved. Deconvolved Hpol waveforms are cross-correlated with each other, with a $10 \mathrm{~ns}$ time window applied around the peak of each one to reduce the bias from noise. For every antenna pair, the correlation coefficient as function of hypothetical TDOA is obtained, and the reconstructed direction is the one which TDOAs give the highest sum of correlation coefficients over all pairs, found by grid search, expressed in zenith $\theta$ (or elevation) and azimuth angles $\phi$.

The result of calibrated reconstruction is shown in Fig. 2 with good agreement, but misreconstruction happened at region around elevation angle below $2^{\circ}$ and azimuth of 5-40 toward higher elevation at $3-5^{\circ}$. One possible cause is that the direct pulses are interfered with the reflected signals from the ground and objects around, as found by HCR and other TAROGE stations $[6,7]$. Such effect can be alleviated later, by mapping and subtracting the response of signal propagation at different angles through more scans with drone pulser, for example, using the technique described in TAROGE analysis [6]. If the biased region is excluded, TAROGE-M has an angular resolution of $0.2^{\circ}$ in azimuth and $0.3^{\circ}$ in elevation. 

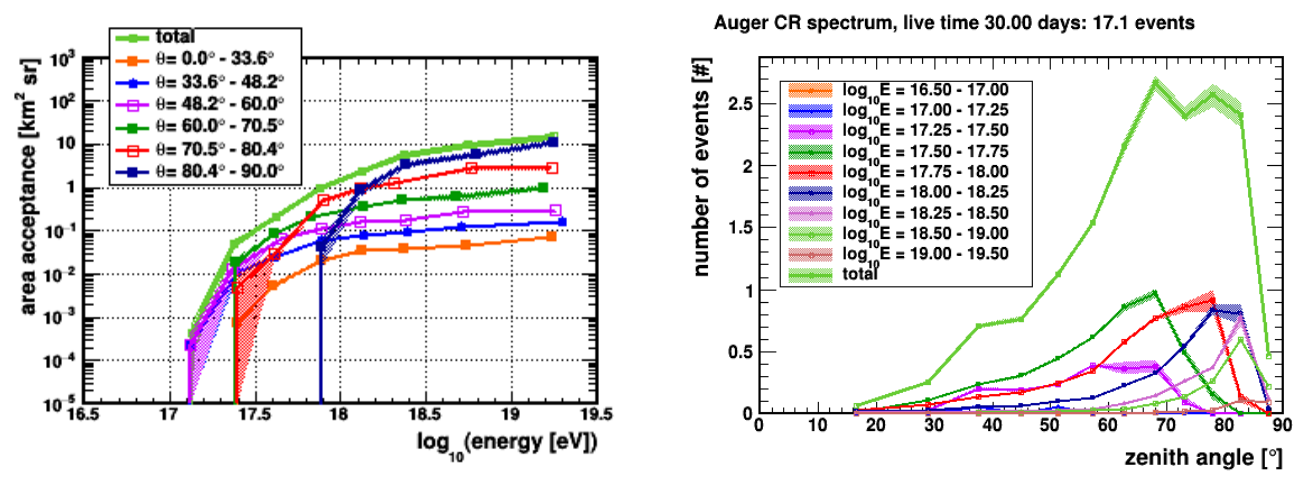

Figure 3: Left: expected cosmic-ray area acceptance of TAROGE-M station versus energy for different zenith angles. Right: expected angular distribution of cosmic ray events, assuming Auger CR spectrum [17] and 30-day exposure. The shaded area shows the uncertainty due to shower-by-shower variation.

\section{Cosmic Ray Search and Detection Simulation}

\subsection{Simulation and Expected Event Distribution}

To estimate the event rate and angular distribution of cosmic rays, Monte-Carlo simulation used in $[7,12]$ was modified for TAROGE-M, while the neutrino acceptance was estimated previously [4]. Radio emission of proton-initiated air showers is simulated with CoREAS [16], and a set of 2,041 showers that were originally for HCR station [7] are used as an approximation, as both stations have similar geomagnetic field but receive the electric field at $30 \mathrm{~m}$ altitude rather than at $2700 \mathrm{~m}$ for TAROGE-M. These showers have energies spanning $30 \mathrm{PeV}$ and $30 \mathrm{EeV}$ and angles over all forward-coming directions to the station. The simulated electric field is convolved with the receiver response (Sec. 3.1) to generate received waveforms, and then passed to simulation of SST trigger as described in Sec. 2 to get detection efficiency. Cosmic ray flux measured by Pierre Auger Observatory [17] is used to calculate the event rate.

The expected cosmic-ray area acceptance and angular distribution of TAROGE-M are shown in Fig. 3, assuming $60 \mathrm{mV}(\sim 4 \sigma)$ trigger threshold. The detection threshold is around $300 \mathrm{PeV}$, and the majority of events come from zenith angle of $60-85^{\circ}$ and energy of $300 \mathrm{PeV}$ to $3 \mathrm{EeV}$, with total 17 events expected in 30 days.

\subsection{Cosmic ray search and background rejection}

A preliminary search for UHE cosmic-ray signals is conducted on the full 30-day data in 2020. There are 1,257, 122 recorded RF-trigger events, where the majority of them are high-wind events (see Sec. 2) with common characteristics that one channel (mostly the veto one) has particularly strong impulse than others and usually has high power at stopband. On the other hand, air-shower signals are expected to be relatively rare, Hpol-dominated, and broadband with higher power at lower frequencies, and of similar strength between receiver channels.

Given the above properties, the following event selection criteria are implemented in the online filtering (in Sec. 2), with the threshold set empirically based on forced-triggered events. The power at $180-240 \mathrm{MHz}$ and $280-330 \mathrm{MHz}$ of an event is defined as its lower passband power, and a threshold of $3.6 \mathrm{~dB}$ on the averaged $\mathrm{Hpol}$ to Vpol power ratio was required. This is a powerful cut to 


\begin{tabular}{|c|r|c|c|r|r|r|}
\hline run & event & timestamp (UTC) & $\begin{array}{c}\text { avg. X-cor } \\
\text { coefficient }\end{array}$ & azimuth $\left(^{\circ}\right)$ & zenith $\left(^{\circ}\right.$ ) & $\begin{array}{c}\text { H/V power } \\
\text { ratio }\end{array}$ \\
\hline 25 & 54906 & $2020 / 01 / 3105: 45: 10$ & 0.81 & -62.2 & 25.5 & 2.4 \\
26 & 50916 & $2020 / 02 / 0717: 11: 37$ & 0.88 & 46.7 & 66.6 & 6.4 \\
26 & 68712 & $2020 / 02 / 0804: 36: 31$ & 0.84 & -14.8 & 42.7 & 4.7 \\
26 & 244803 & $2020 / 02 / 1020: 33: 35$ & 0.80 & 14.8 & 78.5 & 3.3 \\
28 & 69900 & $2020 / 02 / 2222: 51: 36$ & 0.86 & 6.1 & 49.7 & 4.8 \\
\hline
\end{tabular}

Table 1: Summary of cosmic-ray candidate events found in TAROGE-M 2020 data.
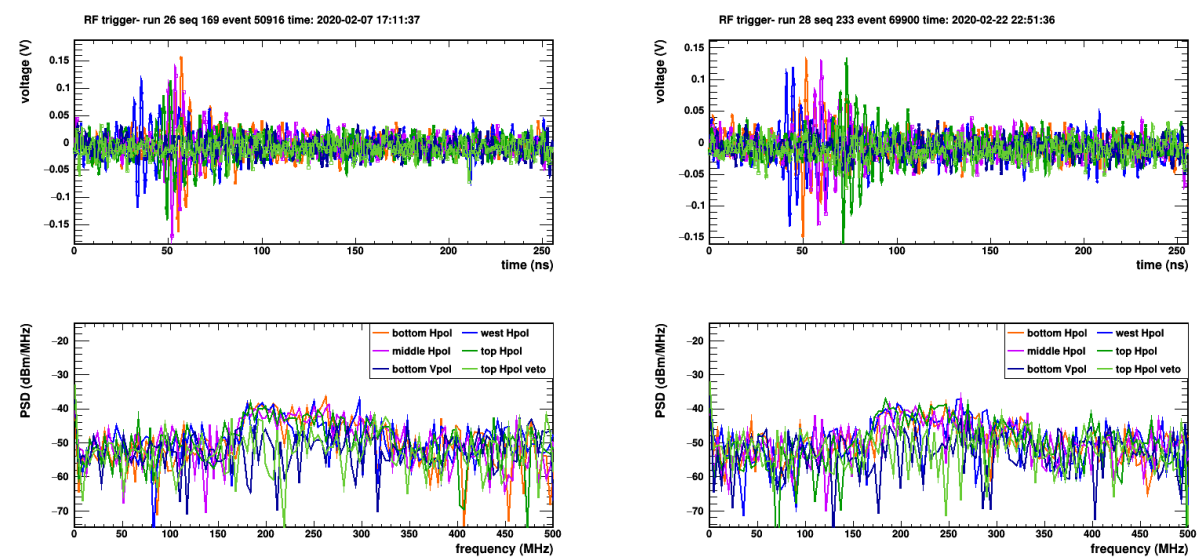

Figure 4: received waveforms (top panels) and power spectra (bottom) of two cosmic-ray candidates.

reject unpolarized thermal noise and select CR. To reject potential satellite noise at $240-280 \mathrm{MHz}$, the Hpol-averaged power ratio of the lower passband to this band higher than $4 \mathrm{~dB}$ is required . An event is rejected as high-wind noise if any of channel has power ratio of lower passband to $130-150 \mathrm{MHz}$ band lower than $1.4 \mathrm{~dB}$ (stopband leakage), or if the difference of lower passband power between any two Hpol channels is $7.5 \mathrm{~dB}$ (channel similarity). Lastly, lower passband power ratio of $\mathrm{Hpol}$ average to veto channel more than $1 \mathrm{~dB}$ is required to reject noise from the back side.

In offline analysis, all events are reconstructed (as in Sec. 3.2 but without deconvolution) assuming plane wave, and events are selected if the average cross-correlation coefficient (between $[0,1])>0.7$ and at the front side of station, to further suppress high-wind events. A temporal cluster cut on selected events requires that a candidate should have no adjacent events within time window of \pm 600 seconds. After all selections are applied, 5 cosmic-ray candidates are identified (Table. 1), and two of them are shown in Fig. 4. The zenith distribution is consistent with the simulation, but the number is lower than the expected one of 17 , as the cuts applied here are not optimized yet. But the ratio of the observed event rate to that of HCR with similar cuts is consistent with what simulation predicted. The efficiency estimate and the optimization of selection cuts are in progress. Template matching will also be used in CR search as in [3, 12]. 


\section{Future Works}

The primary task of TAROGE-M upgrade is to fulfil the full-time operation in summer for fully taking its advantage, so problematic power components will be replaced with military-grade ones. Two more Hpol channels will be installed to improve the angular resolution of event reconstruction. We also plan to install long-range Wi-Fi link from Mt. Melbourne to JBG station for broader data bandwidth, and a ground-based pulser to monitor the reconstruction performance below the horizon over the operation. More stations are expected to be built on the mountain in the next couple of years to increase the area acceptance for confirming the discovery of ANITA's anomalous events.

\section{References}

[1] F. Schröder, Prog. Part. Nucl. Phys. 93 (2017) 1.

[2] P. Gorham et al., Phys. Rev. Lett. 121 (2018) 161102.

[3] P. Gorham et al., Phys. Rev. Lett. 126 (2021) 071103.

[4] J. Nam et al., PoS(ICRC2019)967.

[5] S. Wissel et al., JCAP 11 (2020) 065.

[6] Y. Chen et al., PoS(ICRC2021)263.

[7] S. Wang et al., PoS(ICRC2019)462; also PoS(ICRC2017)358.

[8] S. Kleinfelder, E. Chiem, and T. Prakash, Proc. of 2014 IEEE NSS/MIC (2014) 1.

[9] A. Anker et al., Advances in Space Research 64 (2019) 2595.

[10] A. Romero-Wolf et al., Astropar. Phys. 60 (2015) 72.

[11] Pix4D, https://www.pix4d.com/product/pix4dmapper-photogrammetry-software

[12] S. Barwick et al., Astropar. Phys. 90 (2017) 50.

[13] E. Polisensky, Long Wavelength Array (LWA) Memo Series 111.

[14] High-Frequency Structure Simulator (HFSS) 15.0.3 software, by Ansoft Corporation.

[15] C. Kuo et al., PoS(ICRC2021)283.

[16] T. Huege, M. Ludwig, and C. James, AIP Conference Proceedings 1535 (2013) 128.

[17] V. Verzi et al. (the Pierre Auger Collaboration), PoS(ICRC2019)450. 


\section{Acknowledgements}

We wish to thank the supports from Taiwan Ministry of Science and Technology (MOST) and from Korea Polar Research Institute (KOPRI), and the great logistics and field support from on-ice Jang Bogo station crews in 2019-2020 season. We are grateful to the U.S. National Science Foundation-Office of Polar Programs, the U.S. National Science Foundation-Physics Division (grant NSF-1607719) for supporting the ARIANNA array at Moore's Bay, and NSF grant NRT 1633631. We acknowledge funding from the German research foundation (DFG) under grants GL 914/1-1 and NE 2031/2-1m, the Taiwan Ministry of Science and Technology, the Swedish Government strategic program Stand Up for Energy, MEPhI Academic Excellence Project (Contract No. 02.a03.21.0005) and the Megagrant 2013 program of Russia, via agreement 14.12.31.0006 from 24.06.2013.

\section{Full Authors List: TAROGE and ARIANNA Collaboration}

\section{TAROGE Collaboration}

Pisin Chen ${ }^{1,2,3}$, Yaocheng Chen ${ }^{1,2}$, Jian-Jung Huang ${ }^{1,2}$, Ming-Huey Alfred Huang ${ }^{4}$, Chung-Yun Kuo ${ }^{1,2}$, Chung-Hei Leung ${ }^{1,2}$, TsungChe Liu ${ }^{1,5}$, Jiwoo Nam ${ }^{1,2}$, Yu-Shao Jerry Shiao ${ }^{1}$ Min-Zu Wang ${ }^{1,2}$, Shih-Hao Wang ${ }^{1,2}$, Yu-Hsin Wang ${ }^{1,2}$

${ }^{1}$ Leung Center for Cosmology and Particle Astrophysics, National Taiwan University, No. 1, Sec. 4, Roosevelt Rd., Taipei 10617, Taiwan, R.O.C.

${ }^{2}$ Department of Physics, National Taiwan University, No. 1, Sec. 4, Roosevelt Rd., Taipei 10617, Taiwan, R.O.C.

${ }^{3}$ Kavli Institute for Particle Astrophysics and Cosmology, SLAC National Accelerator Laboratory, Stanford University, Stanford, CA 94305, U.S.A.

${ }^{4}$ Department of Energy Engineering, National United University, Miaoli, Taiwan, R.O.C.

${ }^{5}$ Department of Electrophysics, National Yang Ming Chiao Tung University, Hsinchu 300, Taiwan, R.O.C.

${ }^{6}$ National Nano Device Laboratories, Hsinchu 300, Taiwan, R.O.C.

\section{ARIANNA Collaboration}

Astrid Anker ${ }^{1}$, Pierre Baldi ${ }^{2}$, Steven W. Barwick ${ }^{1}$, Jakob Beise ${ }^{3}$, Hans Bernhoff ${ }^{4}$, Dave Z. Besson ${ }^{5}, 6$, Nils Bingefors ${ }^{3}$, Daniel García Fernández $^{7,8}$, Geoffrey Gaswint ${ }^{1}$, Christian Glaser ${ }^{3}$, Allan Hallgren ${ }^{3}$, Jordan C. Hanson ${ }^{9}$, Spencer R. Klein ${ }^{10}$, Stuart A. Kleinfelder ${ }^{11}$, Robert Lahmann ${ }^{8}$, Jiayi Liu ${ }^{1}$, Mitchell Magnuson ${ }^{5}$, Stephen McAleer ${ }^{2}$, Anna Nelles ${ }^{7,8}$, Alexander Novikov ${ }^{5,6}$, Manuel P. Paul ${ }^{1}$, Christopher Persichilli ${ }^{1}$, Ilse Plaisier ${ }^{7,8}$, Ryan Rice-Smith ${ }^{1}$, Joulien Tatar ${ }^{13}$, Christoph Welling ${ }^{7,8}$, Leshan Zhao ${ }^{1}$

${ }^{1}$ Department of Physics and Astronomy, University of California, Irvine, CA 92697, USA.

${ }^{2}$ Department of Information and Computer Science, University of California, Irvine, CA 92697, USA.

${ }^{3}$ Uppsala University Department of Physics and Astronomy, Uppsala SE-752 37, Sweden.

${ }^{4}$ Uppsala University Department of Engineering Sciences, Division of Electricity, Uppsala SE-752 37, Sweden.

${ }^{5}$ Department of Physics and Astronomy, University of Kansas, Lawrence, KS 66045, USA.

${ }^{6}$ National Research Nuclear University MEPhI (Moscow Engineering Physics Institute), Moscow 115409, Russia. ${ }^{7}$ DESY, 15738 Zeuthen, Germany.

${ }^{8}$ ECAP, Friedrich-Alexander Universität Erlangen-Nürnberg, 91058 Erlangen, Germany.

${ }^{9}$ Whittier College Department of Physics, Whittier, CA 90602, USA.

${ }^{10}$ Lawrence Berkeley National Laboratory, Berkeley, CA 94720, USA.

${ }^{11}$ Department of Electrical Engineering and Computer Science, University of California, Irvine, CA 92697, USA. ${ }^{13}$ Research Cyberinfrastructure Center, University of California, Irvine, CA 92697, USA. 